\title{
Influence of scission neutrons on the prompt fission neutron spectrum calculations
}

\author{
Olivier Serot $^{\mathrm{a}}$, Olivier Litaize, and Abdelaziz Chebboubi \\ CEA, DEN, DER, SPRC, 13108 Saint Paul lez Durance, France
}

\begin{abstract}
The calculation of the Prompt Fission Neutron Spectrum (PFNS) was performed using the FIFRELIN Monte Carlo code simulating the de-excitation of the whole fission fragments. This de-excitation is governed by the Hauser-Feshbach statistical model, which has the advantage to take into account the conservation laws for the energy, spin and parity of the initial and final states. In this way, the competition between prompt neutron and prompt gamma emission can be properly accounted for. Assuming that the prompt neutron emission comes only from an evaporation process of the fully accelerated fission fragments, our calculations are not able to reproduce satisfactorily the experimental data. In this context, we have added an additional source of neutrons that may arise during the sudden rupture of the neck (the so-called scission neutrons). Applied in the case of the spontaneous fission of ${ }^{252} \mathrm{Cf}$, our PFNS calculations show a very good agreement with the Mannhart evaluation by accounting for a $2 \%$ scission neutron contribution.
\end{abstract}

\section{Introduction}

It has been shown in the past that the Prompt Fission Neutron Spectrum (PFNS) is not calculated with enough accuracy in comparison with experimental data if all prompt neutrons are assumed to be evaporated from the fully accelerated fission fragments (see [1] and references therein). As suggested by Kornilov [2], an additional neutron source is probably needed, which could be the so-called 'scission neutrons', i.e. neutrons emitted at the scission point. There are various strong arguments in favor of the existence of scission neutrons. In particular, we know that various ternary light charged particles can be emitted at the scission point: ${ }^{1} \mathrm{H},{ }^{2} \mathrm{H},{ }^{3} \mathrm{H},{ }^{4} \mathrm{He}, \ldots$ up to $\mathrm{A} \sim 40$ [3]. 'Ternary neutrons' (scission neutrons) should therefore also exist and could be even the most produced ternary particle because no Coulomb barrier has to be overcome for their emission, meaning that scission neutrons require less energy to be emitted than the other ternary charged particles. Nevertheless, due to the difficulty to distinguish the 'evaporated neutrons' from the 'scission neutrons', experimental data related to the scission neutrons characteristics (multiplicity, energy) are very contradictory. For example, in the case of the spontaneous fission of ${ }^{252} \mathrm{Cf}$, Bowman [4] has found a scission neutron contribution of about $10 \%$, while BudtzJorgensen [5] claimed that their contribution is less than $1 \%$. More recently, a contribution of $8 \%$ was found by Chietera [6]. From the theoretical point of view, the socalled 'sudden approximation' model proposed initially by Fuller [7], is a convincing description of the ternary particle emission process. Applying this model to the scission neutron emission, Capote [8] found recently a contribution of about $19 \%$ in the case of ${ }^{252} \mathrm{Cf}(\mathrm{sf})$. Nevertheless, as mentioned by the authors, this result

a e-mail: olivier.serot@cea.fr represents an upper limit since the possible re-absorption of unbound neutrons is not taken into account in their calculations.

In the present work, our aim is to calculate PFNS by using the FIFRELIN Monte Carlo code which simulates the evaporation of prompt neutrons from the fully accelerated Fission Fragment (FF) and by adding a scission neutron component. We focus on the ${ }^{252} \mathrm{Cf}$ spontaneous fission, for which PFNS is accurately described by the Mannhart evaluation [9].

The first section describes our 'Reference calculation' performed with FIFRELIN without scission neutron. Then, in the second part, we explain how scission neutrons are incorporated within the 'three sources' model (following the denomination proposed by Kornilov [1]). In the last section, results obtained with this model are presented.

\section{2. 'Reference Calculation' (without scission neutron)}

The 'FIFRELIN' Monte-Carlo code developed at CEACadarache aims, on one hand, at calculating post-fission observables (spectra and multiplicities of the prompt neutron and gamma particles, energies released in fission, ... ) and on the other hand, at investigating correlations between fission observables. Hence, it is possible to access nuclear data useful for both nuclear reactor applications and our understanding of the fission process. The code simulates the de-excitation of the FF by using the HauserFeshbach formalism [10] and the procedure proposed by Becvar [11]. In this way, the competition between prompt neutron and prompt gamma emission is properly taken into account. The conservation laws for the energy, spin and parity of the initial and final states are also respected. All the details related to the code can be found in Refs. [12-14]. Since for this 'Reference Calculation' no

(C) The Authors, published by EDP Sciences. This is an Open Access article distributed under the terms of the Creative Commons Attribution License 4.0 (http://creativecommons.org/licenses/by/4.0/). 


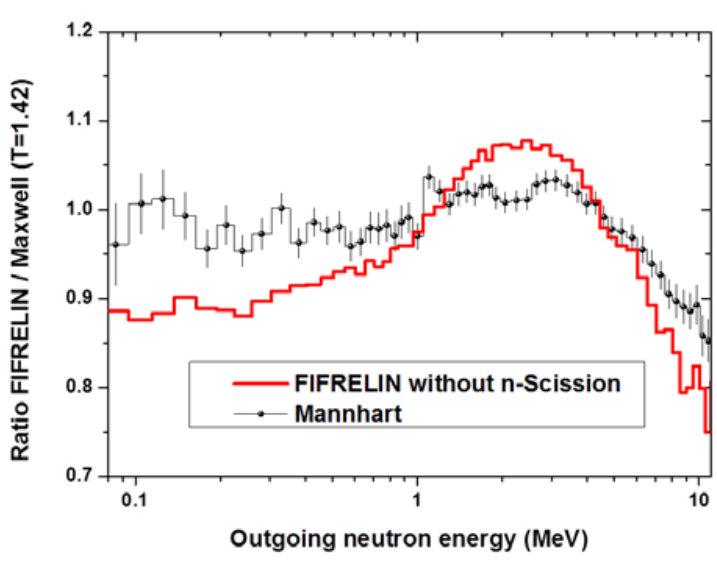

Figure 1. PFNS calculated using FIFRELIN code without scission neutron ('Reference calculation'). This PFNS is plotted as a ratio with a Maxwellian $(\mathrm{T}=1.42 \mathrm{MeV})$ and is compared to the Mannhart evaluation [9].

scission neutron is emitted, prompt neutrons result from a pure evaporation process. The main ingredients used for the calculation $(\gamma$-strength function, neutron transmission coefficients, level density parameter, shell corrections and so on) are described in Ref. [15].

The input data needed for the calculation (pre-neutron mass yield, pre-neutron kinetic energy distribution as a function of mass) are taken from Ref. [16]. Our 'Reference Calculation' is plotted in Fig. 1. As recommended by Litaize et al. in Ref. [15], we used the MengoniNakajima shell corrections (involved in the level density parameter), which allows a better description of the PFNS. Nevertheless, a slight underestimation below $1.5 \mathrm{MeV}$ and above $4 \mathrm{MeV}$ is still persisting, while in between 1.5 and $4 \mathrm{MeV}$ the calculated PFNS shows a slight overestimation. Note that the average prompt neutron multiplicities for the light and the heavy fragment measured by Göök [17] $\left(<v_{L}\right\rangle=2.09$ and $\left.\left\langle v_{H}\right\rangle=1.67\right)$ are nicely reproduced.

\section{The 'three sources' model revisited}

The 'three sources' model has been initially proposed by Kornilov [2]. A Watt spectrum was adopted to describe the evaporated neutron components and the ratio of the neutron multiplicity between the light and the heavy fragment was fixed for simplicity at $1 / 2$. In addition, two arbitrary components were introduced to describe the scission neutron spectrum. by:

In the present work, we have tried to improve the model

- calculating the evaporated neutron spectra and multiplicities with the FIFRELIN code,

- determining the scission neutron spectrum from physical grounds,

- conserving in our calculations the total prompt neutron multiplicity $\left(\left\langle v_{T o t}\right\rangle=3.76\right)$ and the total available excitation energy $(<T X E>=34.2 \mathrm{MeV}$, taken from [18]).

\subsection{Description of the model}

Prompt neutrons are assumed to be emitted from three different sources. The total PFNS is therefore described by three different components which are described below.
- 1st component. When no scission neutron is emitted (with a probability equal to: $1-b^{S C}$ ), the compound nucleus $\mathrm{A}$ (here, $\mathrm{A}=252$ ) is fissioning into two fragments having an average excitation energy noted $E_{L}^{*}$ and $E_{H}^{*}$ for the light $(\mathrm{L})$ and the heavy $(\mathrm{H})$ fragment respectively. Prompt neutrons are assumed to be evaporated from the fully accelerated FF with an average multiplicity noted: $\left\langle v_{L}^{E v a}\left(E_{L}^{*}\right)>\right.$ and $<v_{H}^{E v a}\left(E_{H}^{*}\right)>$ for the light and heavy fragment (' $E v a$ ' stands for 'Evaporated'). $b^{S C}$, which represents the relative scission neutron emission probability, is a free parameter in our model.

- 2nd component. In the case where one scission neutron is emitted, the two fragments have less excitation energy. We note $E^{S C}$ the energy needed to emit one scission neutron. If this energy is equally removed from the two fragments, then the average excitation energy available for the light and the heavy fragment are respectively: $E_{L}^{*}-0.5 E^{S C}$ and $E_{H}^{*}-$ $0.5 E^{S C}$

- 3rd component. The third neutron component corresponds to the scission neutrons. Their multiplicity is simply given by: $\left\langle v^{S C}\right\rangle=b^{S C}\left\langle v_{T o t}\right\rangle .\left\langle v^{S C}\right\rangle$ is assumed to be equally shared between the light and the heavy fragment, so that: $\left\langle v_{L}^{S C}\right\rangle=\left\langle v_{H}^{S C}\right\rangle=$ $0.5<v^{S C}>$.

The total prompt neutron multiplicity from the light $(\mathrm{L})$ and the heavy $(\mathrm{H})$ fragment $\left(\left\langle v_{L, H}^{T o t}\right\rangle\right)$ is then obtained by summing the three components:

$$
\begin{aligned}
<v_{L, H}^{T o t}>= & \left(1-b^{S C}\right)<v_{L, H}^{E v a}\left(E_{L, H}^{*}\right)> \\
& +b^{S C}<v_{L, H}^{E v a}\left(E_{L, H}^{*}-0.5 E^{S C}\right)>+0.5 b^{S C}<v_{T o t}>
\end{aligned}
$$

with $\left.\left\langle v_{L}^{T o t}\right\rangle+<v_{H}^{T o t}\right\rangle=3.76$. The corresponding PFNS from the light and the heavy fragment is given by:

$$
\begin{aligned}
<v_{L, H}^{T o t}>\chi_{L, H}^{T o t}= & \left(1-b^{S C}\right)<v_{L, H}^{E v a}\left(E_{L, H}^{*}\right)>\chi_{L, H}^{E v a}\left(E_{L, H}^{*}\right) \\
& +b^{S C}<v_{L, H}^{E v a}\left(E_{L, H}^{*}-0.5 E^{S C}\right)> \\
& \times \chi_{L, H}^{E v a}\left(E_{L, H}^{*}-0.5 E^{S C}\right) \\
& +0.5 b^{S C}<v_{T o t}>\chi^{S C}
\end{aligned}
$$

The total PFNS can be then deduced by: $\chi^{\text {Tot }}=$ $\left(1 / v_{T o t}\right)\left(<v_{L}^{T o t}>\chi_{L}^{T o t}+<v_{H}^{T o t}>\chi_{H}^{T o t}\right)$. Several quantities are needed for the $\chi^{\text {Tot }}$ calculation: $b^{S C}, E^{S C}, E_{L}^{*}$, $E_{H}^{*},<v_{L}^{E v a}\left(E_{L}^{*}\right)>,<v_{H}^{E v a}\left(E_{H}^{*}\right)>, \chi_{L}^{E v a}\left(E_{L}^{*}\right), \chi_{H}^{E v a}\left(E_{H}^{*}\right)$ and $\chi^{S C}$. The way how each quantity was calculated is explained in the following sub-sections.

\subsection{Scission neutron spectrum: $\chi^{s c}$}

We know that all ternary particles (except for ternary $\alpha$-particles, due to the presence of ${ }^{5} \mathrm{He}$ ternary particles) have a Gaussian Kinetic Energy (KE) distribution type (see for example Ref. [3] and references therein). For charged ternary particles, the average $\mathrm{KE}$ is strongly correlated to the Coulomb barrier. So, for scission neutrons (with no Coulomb barrier), the average $\mathrm{KE}$ is expected to be close to zero. In the present work, $\chi^{S C}$ is described by a Gaussian with an average energy of $0.25 \mathrm{MeV}$, close to the value suggested by Capote et al. in Ref. [19]. 


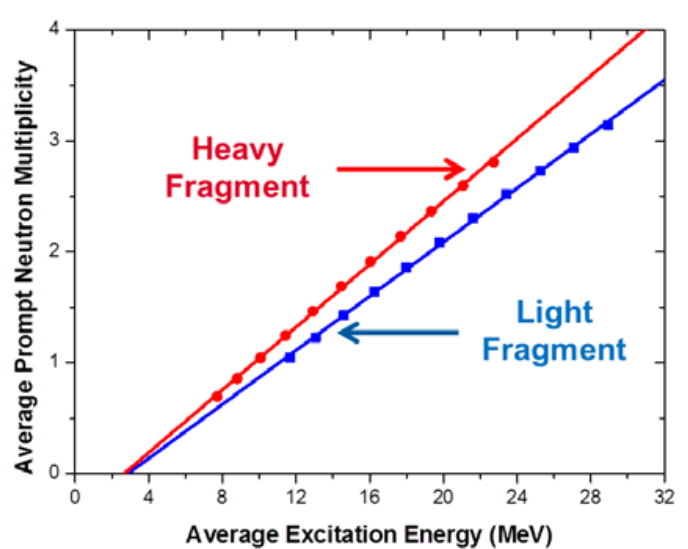

Figure 2. Average neutron multiplicity as a function of the average available excitation energy. A clear linear correlation is observed for both the heavy fragment (red curve) and the light one (blue curve).

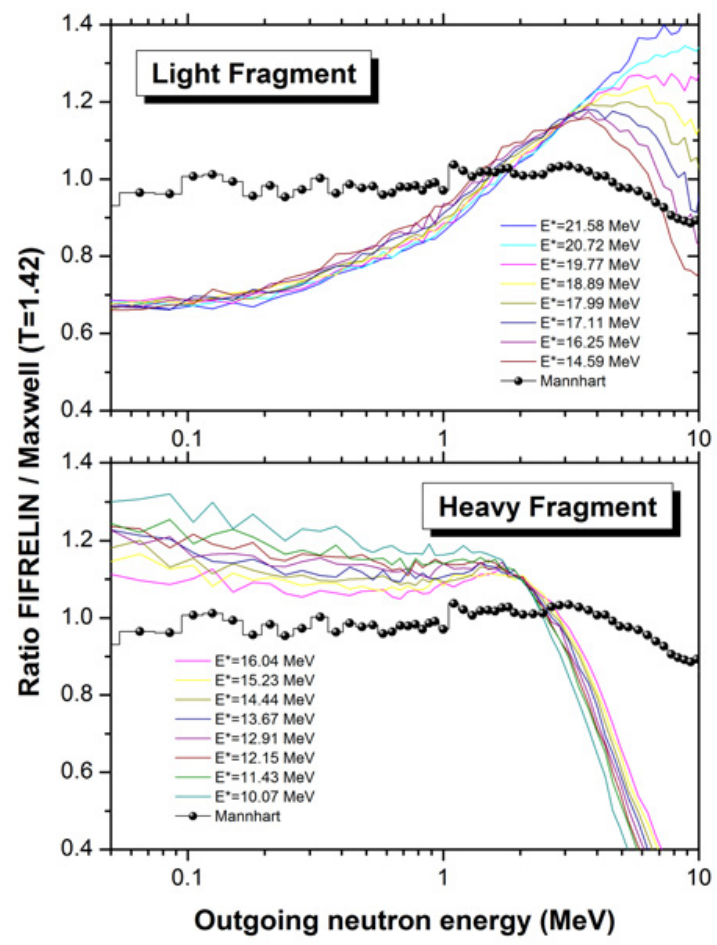

Figure 3. PFNS calculated at various average excitation energies, for the light (top) and the heavy (bottom) fragment.

\subsection{Evaporated neutron spectra and multiplicities}

The evaporated prompt neutron spectra and multiplicities must be calculated (with the FIFRELIN code) for several average excitation energies for both light and heavy fragments. To do that, a constant shift of the fission fragment average kinetic energy is applied for all masses and is used as a new input data for FIFRELIN. For example, a shift of $+4 \mathrm{MeV}$ leads to an average excitation energy for the light fragment equal to $16.25 \mathrm{MeV}$ (instead of $19.77 \mathrm{MeV}$ for the 'reference calculation') and an average excitation energy for the heavy fragment equal to $11.43 \mathrm{MeV}$ (instead of $14.44 \mathrm{MeV}$ for the 'reference calculation'). In this example, the average evaporated neutron multiplicities is obviously modified: 1.64 and 1.25 for the light and heavy fragment respectively (instead of
Table 1. Survey of the parameters used to get the total PFNS shown in Fig. 4.

\begin{tabular}{cccccc}
\hline $\begin{array}{c}b^{S C} \\
(\%)\end{array}$ & $\begin{array}{c}E^{S C} \\
(\mathrm{MeV})\end{array}$ & $\begin{array}{c}E_{L}^{*} \\
(\mathrm{MeV})\end{array}$ & $\begin{array}{c}E_{H}^{*} \\
(\mathrm{MeV})\end{array}$ & $v_{L}^{\text {Tot }}$ & $v_{H}^{\text {Tot }}$ \\
\hline 2 & 4.7 & 21.48 & 12.72 & 2.30 & 1.46 \\
\hline
\end{tabular}

2.08 and 1.69 for the 'Reference calculation'). Repeating this procedure for several different shifts leads to Figs. 2 and 3. In Fig. 2, the average neutron multiplicity is plotted as a function of the average excitation energy. A clear linear correlation was found for both light and heavy fragments:

$$
<v_{L, H}^{E v a}\left(E_{L, H}^{*}\right)>=a_{L, H}+b_{L, H} E_{L, H}^{*}
$$

The following coefficients were found: $a_{L}=-0.350$, $b_{L}=0.122 \mathrm{n} / \mathrm{MeV}, a_{H}=-0.377, b_{H}=0.142 \mathrm{n} / \mathrm{MeV}$.

The evaporated neutron spectra are shown (as a ratio to a Maxwellian with $\mathrm{T}=1.42 \mathrm{MeV}$ ) for various excitation energies for the light fragment (top of the Fig. 3) and for the heavy one (bottom of the Fig. 3). It is interesting to note that for the light fragment, the available excitation energy does not impact PFNS below about $3 \mathrm{MeV}$ but has a strong influence above. For the heavy fragment, the opposite situation is observed: the available excitation energy does not impact PFNS above $2 \mathrm{MeV}$ but has a strong influence below.

\subsection{Energy cost for scission neutron emission}

Replacing Eq. (3) into Eq. (1) allows us to determine $E_{L, H}^{*}$ :

$$
\begin{aligned}
E_{L, H}^{*}= & \frac{1}{b_{L, H}}\left(<v_{L, H}^{T o t}>-a_{L, H}\right. \\
& \left.+\frac{b^{S C}}{2}\left(b_{L, H} E^{S C}-<v_{T o t}>\right)\right)
\end{aligned}
$$

Keeping in mind that the total excitation energy must be conserved $\left(T X E=E_{L}^{*}+E_{H}^{*}\right)$, a very simple relation between $E^{S C}$ and $b^{S C}$ can be deduced:

$$
\begin{aligned}
E^{S C}= & \frac{1}{b^{S C}}\left[T X E-\frac{<v_{L}^{T o t}>}{b_{L}}-\frac{<v_{H}^{T o t}>}{b_{H}}+\frac{a_{L}}{b_{L}}+\frac{a_{H}}{b_{H}}\right] \\
& +0.5<v_{T o t}>\left(\frac{1}{b_{L}}+\frac{1}{b_{H}}\right) .
\end{aligned}
$$

\section{Results}

The procedure for the calculation consists in choosing $\left\langle v_{L}^{T o t}\right\rangle$ and $b^{S C}$ which are therefore considered in our model as two free parameters. Then $\left\langle v_{H}^{T o t}\right\rangle$ is given by $\left\langle v^{T o t}>-\left\langle v_{L}^{T o t}>\right.\right.$. Equation (5) allows the determination of $E^{S C}$ (with TXE=34.2 MeV), so that the average excitation energies $E_{L, H}^{*}$ can be deduced from Eq. (4). Then $\chi_{L, H}^{E v a}\left(E_{L, H}^{*}\right)$ and $\chi_{L, H}^{E v a}\left(E_{L, H}^{*}-0.5 E^{S C}\right)$ must be calculated using the FIFRELIN code. Weighting each spectrum by their multiplicity according to Eq. (2) allows the determination of the total PFNS $\chi^{T o t}$. In this way, $\chi^{T o t}$ can be compared with the Mannhart evaluation. If the agreement is not satisfactory, other values for $\left\langle v_{L}^{T o t}\right\rangle$ and $b^{S C}$ have to be chosen.

As shown in Fig. 4, a very nice agreement compared to Mannhart evaluation can be found. The parameters 


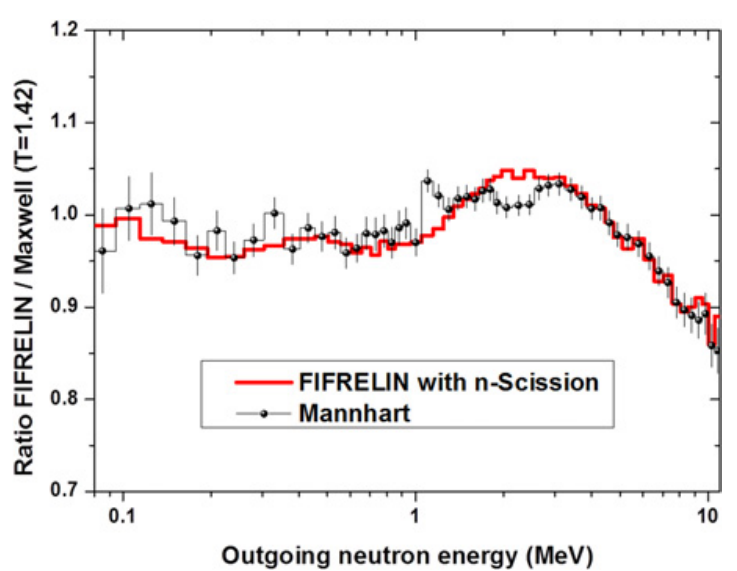

Figure 4. PFNS calculated with the 'three sources' model and using the parameters given in Table 1 . A very nice agreement with the Mannhart evaluation can be seen.

used to get this result are summarized in Table 1 . A scission neutron contribution of only $2 \%$ is needed to get such a good agreement (very close to the value suggested in Ref. [19]). $E^{S C}$ was found to be about $5 \mathrm{MeV}$, which is close to the value calculated by Carjan in Ref. [20]. Nevertheless, an overestimation of the total prompt neutron multiplicity from the light fragment $\left(\left\langle v_{L}^{T o t}\right\rangle=\right.$ 2.3) compared to the experiment $\left(\left\langle v_{L}^{\text {Exp }}>=2.09\right.\right.$, Ref. [17]) is required to reach this agreement.

\section{Conclusion}

In the present work, the 'three sources' model proposed by Kornilov has been revisited. The evaporated neutrons are simulated with the FIFRELIN Monte Carlo code and the scission neutrons are represented by a Gaussian (with an average energy of $0.25 \mathrm{MeV}$ ). All components needed for the PFNS calculation are deduced in order to respect the total prompt neutron multiplicity (3.76) and the average total excitation energy (34.2 MeV). A very nice agreement with the Mannhart evaluation was obtained by using a low scission neutron component of $2 \%$.

\section{References}

[1] N. Kornilov, Fission Neutrons (Experiments, Evaluation, Modeling and Open Problems) (Springer International, Switzerland, 2015)

[2] N. Kornilov, A.B. Kagalenko, S.V. Poupko et al., Nucl. Phys. A686, 187-203 (2001)

[3] C. Wagemans, The Nuclear Fission Process (CRC Press, 1991)

[4] H.R. Bowman, et al., Phys. Rev. 126, 2120 (1962)

[5] C. Budtz-Jorgensen and H.H. Knitter, Nucl. Phys. A490, 307 (1988)

[6] A. Chietera, PhD thesis, University of Strasbourg (2015)

[7] R.W. Fuller, Phys. Rev. 126, 684 (1962)

[8] R. Capote, N. Carjan, and S. Chiba, Phys. Rev. C93, 024609 (2016)

[9] W. Mannhart, in IAEA Report No. IAEA-TECDOC410, p.158 (1987)

[10] W. Hauser, and H. Feshbach, Phys. Rev. 87, 366 (1952)

[11] F. Becvar, Nucl. Instrum. Meth. Phys. Res. A 417, 434 (1998)

[12] O. Litaize, and O. Serot, Phys. Rev. C82, 054616 (2010)

[13] D. Regnier, O. Litaize, and O. Serot, Computer Physics Communications 201, 19-28 (2016)

[14] O. Litaize, O. Serot, and L. Berge, Eur. Phys. J. A51, 177 (2015)

[15] O. Litaize, O. Serot, L. Thulliez and A. Chebboubi, these proceedings

[16] N. Varapai, et al., Proc. of the Int. Workshop on Nuclear Fission and Fission Product Spectroscopy, Cadarache, France, 2005, edited by H. Goutte et al., AIP Conf. Proc. No. 798 (AIP, New York, 2005), p. 369

[17] A. Göök, F.-J. Hambsch, and M. Vidali, Phys. Rev. C90, 064611 (2014)

[18] P. Heeg, PhD thesis, Darmstadt (1990)

[19] R. Capote et al., Nucl. Data Sheets 131, 1-106 (2016)

[20] N. Carjan, F.-J. Hambsch, M. Rizea, and O. Serot, Phys. Rev. C85, 044601 (2012) 